\title{
Scaling and interaction-assisted transport in graphene with one-dimensional defects
}

\author{
M. Kindermann ${ }^{1}$ \\ 1 School of Physics, Georgia Institute of Technology, Atlanta, Georgia 30332, USA
}

(Dated: March 2010)

\begin{abstract}
We analyze the scattering from one-dimensional defects in intrinsic graphene. The Coulomb repulsion between electrons is found to be able to induce singularities of such scattering at zero temperature as in one-dimensional conductors. In striking contrast to electrons in one space dimension, however, repulsive interactions here can enhance transport. We present explicit calculations for the scattering from vector potentials that appear when strips of the material are under strain. There the predicted effects are exponentially large for strong scatterers.
\end{abstract}

PACS numbers: 72.80.Vp, 71.45.Gm, 72.10.Fk, 73.63.-b

The Coulomb repulsion between electrons can have profound consequences for the scattering of electrons in conductors. In one-dimensional (1D) conductors it dramatically suppresses the conductance through impurities, with a singularity at temperature $T=0$. This is one of the hallmarks of the Luttinger liquid state of interacting electrons in 1D [1]. A particularly intuitive understanding of this effect is due to Matveev, Yue, and Glazman [2, 3]. In their approach interactions cause extra electron scattering from Friedel oscillations, which inhibits transport. Friedel oscillations are density modulations that form when electron waves incident on an impurity interfere with backscattered waves. In $1 \mathrm{D}$ at $T=0$ the Friedel oscillation around an impurity is not integrable over space and the induced scattering amplitude diverges logarithmically in Born approximation.

Friedel oscillations in two dimensions (2D) at nonzero chemical potential can be integrated and have much weaker effects for both, point-like [4-6] and 1D defects [7, 8]. Interactions play a more important role in graphene [9-11] at zero chemical potential ("intrinsic graphene"), which forms a so-called marginal Fermi liquid [12]. There is, for instance, experimental evidence 13. of a singular renormalization of the Fermi velocity 12, 14]. Nevertheless, also in intrinsic graphene scattering from point-like impurities of both, scalar and vector potential character, does not receive any singular corrections from the Coulomb interaction [15, 16] 23].

In this Letter we show that the Coulomb interaction in intrinsic graphene can have a singular impact on scatterers that are extended in one space dimension. A qualitative analysis of the Friedel oscillations at such scatterers reveals the reason: the Friedel oscillation due to an electron with wavevector $k^{\prime}$ scattering with amplitude $r$ from a 1D defect fills a strip of width $\sim 1 / k^{\prime}$. The corresponding exchange potential has support in the same strip, implying a factor $\sim r / k^{\prime}$ in the induced Born scattering amplitude $\delta r^{\mathrm{ex}}$. A second factor $1 / k^{\prime}$ comes from the Coulomb interaction. For energy-independent $r$ the exchange interaction with electrons in all filled states thus results in $\delta r^{\text {ex }} \propto r \int_{\text {filled }} d^{2} k^{\prime} / k^{\prime 2}$. Since in intrinsic graphene at $T=0$ the entire valence band is filled, $\delta r^{\text {ex }}$ is logarithmically divergent. This suggests that when the Coulomb interaction does not cause a Hartree potential it can have the same drastic effects for 1D scatterers in 2D intrinsic graphene as for point defects in 1D conductors.

In contrast to the familiar situation in 1D [1], however, we demonstrate that the Coulomb interaction in graphene can enhance electron transport. This intriguing effect is due to bound states that straddle defects. The exchange interaction of transport electrons with electrons in such bound states opens an additional transport path across the defect, which increases the electric current.

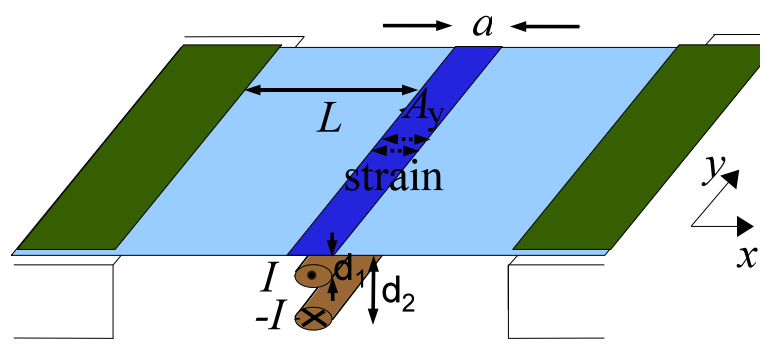

FIG. 1: A vector potential $A_{y}$ in a narrow strip (dark blue) forms a scattering barrier in a sheet of otherwise ballistic graphene (light blue with contacts in green). $A_{y}$ may be induced by strain or, alternatively, by a pair of current-carrying wires (brown).

We exemplify the above effects with the scattering barrier shown in Fig. 1, formed by a vector potential in a narrow strip of an otherwise ballistic sheet of intrinsic graphene. Such vector potentials are induced by strain [17, 18] or by nearby electric currents, see Fig. 1. By symmetry vector potentials in graphene do not produce a Hartree potential. As suggested by the above argument, the Coulomb interaction therefore induces extra scattering whose amplitude diverges at low $T$, when the electron wavelength is much longer than the width $a$ of the barrier and scattering is energy-independent. The effect turns out to be exponentially enhanced for strong barriers, where it strongly impacts transport even at moderate $T$. In addition, the scatterer of Fig. 1 hosts bound 
states that enhance transport through electron exchange as motivated above. As a consequence, the barrier of Fig. 1 1 becomes entirely transparent at $T=0$, in striking contrast to impurities in interacting $1 \mathrm{D}$ conductors that completely block the electric current at $T=0$.

Model: In the setup of Fig. 1 a vector potential

$$
\mathbf{A}=\left(0, A_{y}\right) ; \quad A_{y}=\frac{\tilde{\chi}}{e a}\left[\Theta\left(x+\frac{a}{2}\right)-\Theta\left(x-\frac{a}{2}\right)\right]
$$

$[\Theta(x)$ is the step function] is applied to a sheet of clean, intrinsic graphene that we first assume to be infinite, $L \rightarrow$ $\infty$. The noninteracting Hamiltonian in valley $\gamma$ thus is

$$
H_{\gamma}=v \boldsymbol{\sigma}_{\gamma} \cdot(\boldsymbol{p}-e \boldsymbol{A}) .
$$

Here, $\boldsymbol{\sigma}_{\gamma}=\left(\sigma_{x}, \gamma \sigma_{y}\right)$ is a vector of Pauli matrices, $\boldsymbol{p}$ is the electron momentum, $v$ the electron velocity, and $-e$ the electron charge. The above model has parity symmetry, $\mathcal{P} H \mathcal{P}=H$, where $\mathcal{P} \psi(x, y)=\sigma_{\mathrm{y}} \psi(-x, y)$, and particle-hole $(\mathrm{PH})$ symmetry, $\sigma_{z} H \sigma_{z}=-H$. In intrinsic graphene both symmetries are also respected by electronelectron interactions [24]. Without restriction we assume $\tilde{\chi}>0$. Transport through the barrier at $\tilde{\chi}<0$ is obtained through reflection on the $x$-axis. The above model has been analyzed in Ref. [17] on a noninteracting level, where $\mathbf{A}$ suppresses linear transport exponentially in $\tilde{\chi}$ if $k T \ll \tilde{\chi} v / a$ (we set $\hbar=1$ ). Below we study the effects of the Coulomb repulsion between electrons on this transport problem for short barriers $(\Lambda a \gg 1$, but $\ln \Lambda a \gtrsim 1$, where $\Lambda$ is the bandwidth of graphene).

We assume that the graphene sheet is suspended or lying on an insulating substrate, leaving the Coulomb interaction unscreened with interaction potential $V_{\mathrm{C}}(\mathbf{q})=$ $2 \pi \tilde{r}_{\mathrm{s}} v /|\boldsymbol{q}|$ at interaction parameter $\tilde{r}_{\mathrm{s}}$ and wavevector $\mathbf{q}$
[25]. In the weakly interacting limit $\tilde{r}_{\mathrm{s}} \ll 1$ that we take henceforth the main effect of electron-electron interactions is additional elastic scattering, as in the thoroughly studied 1D problem [2, 3, 19]. Also like in 1D, such scattering is induced by the Hartree-Fock potential $V^{\mathrm{HF}}$ due to Friedel oscillations at the barrier. In addition, $\mathbf{A}$ here induces bound states with an extra contribution to $V^{\mathrm{HF}}$.

The potential $V^{\mathrm{HF}}$ is strongly constrained by the symmetries of the setup. Most importantly, a Hartree potential is precluded by PH-symmetry. Also, the exchange potential $V^{\mathrm{ex}}$ is spin- and valley-diagonal. Transport in both valleys is identical since they are related as $H_{-1}=U_{\mathrm{v}}^{\dagger} H_{1} U_{\mathrm{v}}$, where $U_{\mathrm{v}}=\sigma_{\mathrm{x}}$ commutes with the current $\sigma_{\mathrm{x}}$ through the barrier [26]. We thus set $\gamma=1$.

The compound scatterer composed of $\mathbf{A}$ and $V^{\text {ex }}$ is conveniently described by the transfer matrix of electrons at zero energy $M=\left.T(\infty,-\infty)\right|_{\varepsilon=0}[\underline{6}]$. The $\mathcal{P}_{-}$ and PH-symmetries of our model imply $\sigma_{\mathrm{y}} M \sigma_{\mathrm{y}}=M^{-1}$ and $\sigma_{\mathrm{z}} M \sigma_{\mathrm{z}}=M$, respectively. Together with current conservation $\sigma_{\mathrm{x}} M^{\dagger} \sigma_{\mathrm{x}}=M^{-1}$ they constrain $M$ to take the form $M=\exp \left(\chi \sigma_{\mathrm{z}}\right)$. Low energy transport in the setup of Fig. 1 with weak many-body interactions is thus characterized by a single parameter $\chi$. This reduces our analysis to a calculation of $\chi$. At $\tilde{r}_{\mathrm{s}}=0$ we have $\chi=\tilde{\chi}$.

First order in $\tilde{r}_{\mathrm{s}}$ : To begin with, we calculate the interaction correction to $\tilde{\chi}$ at first order in $\tilde{r}_{\mathrm{s}}$. Computing the correction to $M$ due to scattering from the exchange potential created by the noninteracting electron states in Born approximation we find in the limit $a \ll v / k T$

$$
\chi=\tilde{\chi}-\tilde{r}_{\mathrm{s}} \sinh \tilde{\chi} F(\tilde{\chi})\left[\ln \frac{v}{k T a}-\tilde{\chi}+\mathcal{O}(1)\right]
$$

with the positive function

$$
F(\chi)=\frac{\operatorname{coth} \chi(\cosh 3 \chi-3 \cosh \chi) \arcsin (\tanh \chi)+2 \cosh 2 \chi}{2 \pi}-\cosh \chi \sinh ^{2} \chi
$$

The first order result Eq. (3) manifests itself in a non-analytic temperature-dependence of the conductance through the barrier, which takes the form

$$
G=\frac{4 e^{2}}{h} \frac{k T W \ln 2}{\pi v}\left(1+\sinh \chi \tanh \chi \ln \tanh \frac{\chi}{2}\right) .
$$

In $G$, the logarithmic temperature dependence of the interaction correction to $\chi$ competes with the factor $T$ due to the linear density of states. The predicted non-analytic scale-dependence of $\chi$ is thus more easily observed in the normalized conductance $G_{\mathrm{r}}=G /\left(\left.G\right|_{\tilde{\chi}=0}\right)$ [27].

Due to its logarithmic $T$-dependence the first order correction to $\tilde{\chi}$ may grow large even at $\tilde{r}_{\mathrm{s}} \ll 1$. At the corresponding low temperatures, when $\tilde{r}_{\mathrm{s}} \ln (v / k T a) \gtrsim 1$, the above perturbative calculation looses validity, as in the $1 \mathrm{D}$ case $[2,3]$. The origin of the logarithmic divergence here is, however, different. In 1D the divergence stems from a Friedel oscillation density $\propto 1 / x$ at $T=0$. In the present $2 \mathrm{D}$ case the $T=0$ Friedel oscillation density is $\propto 1 / x^{2}$ and integrable. But the non-locality of $V^{\mathrm{ex}}$ gives rise to the same logarithmic divergencies as in 1D.

$R G$-analysis: We extend our analysis into the regime $\tilde{r}_{\mathrm{s}} \ll 1$, but $\tilde{r}_{\mathrm{s}} \ln (v / k T a) \gtrsim 1$, by a renormalization group (RG) calculation that re-sums the perturbation series in the "leading logarithm" approximation [2, 3]. To this end we successively integrate out shells of wavevectors $\left[k^{\prime}, b k^{\prime}\right]$ with $b>1$ in the order of decreasing $k^{\prime} \ll 1 / a$. For each shell we compute the exchange potential $V_{k^{\prime}, b}^{\mathrm{ex}}$ due to 
the corresponding states. Thereafter we renormalize the transfer matrix for low energy electrons at wavevector $k$ by $V_{k^{\prime}, b}^{\mathrm{ex}}$. The resulting $\chi$ characterizes the wavefunctions in the subsequent shell $[k, b k]$. The logarithmic scaledependence of $\delta \chi$, Eq. (3), allows us to assume $k \ll k^{\prime}$ in this process. Introducing $l=-\ln k^{\prime} a$ we thus find that

$$
\frac{d \chi}{d l}=-r_{\mathrm{s}} \sinh \chi F(\chi),
$$

where $\chi=\tilde{\chi}$ at $l=0$. Here, $r_{\mathrm{s}}$ is a scale-dependent interaction parameter that is renormalized as $d r_{\mathrm{s}} / d l=$ $-r_{\mathrm{s}}^{2} / 4$ [12] with the solution $r_{\mathrm{s}}=\tilde{r}_{\mathrm{s}} /\left(1+\tilde{r}_{\mathrm{s}} l / 4\right)$. Eq. (6) with this $l$-dependent $r_{\mathrm{s}}$ is solved best by introducing $y=\ln \left(1+\tilde{r}_{\mathrm{s}} l / 4\right)$, such that $d \chi / d y=-4 \sinh \chi F(\chi)$.

We start the detailed analysis of Eq. (6) assuming $\tilde{\chi} \gg$ 1. As long as $\chi \gg 1$ we use $\lim _{\chi \rightarrow \infty} F(\chi)=2 / 3 \pi$ to find

$$
\frac{d \chi}{d y}=-\frac{4}{3 \pi} e^{\chi}
$$

Eqs. (6) and (7) make two remarkable predictions: First, the minus signs in both equations imply that the Coulomb interaction reduces the barrier strength. It thus enhances transport through the studied scatterer, in evident contrast with the familiar situation in 1D [1 3 ]. Second, that enhancement is exponentially large in $\chi$ such that for sufficiently large $\tilde{\chi}$ the Coulomb interaction strongly impacts electron transport even at $r_{\mathrm{s}} l \ll 1$.

Both predictions have their origin in bound states. We find that the interaction with the Friedel oscillations due to propagating states decreases the conductance as in 1D. That suppression, however, is overcompensated by bound states which form inside the scattering barrier at $|x|<a / 2$, but leak into the region $|x|>a / 2$. Electrons in bound states have the same probability for being to the left of the barrier as to the right, guaranteed by $\mathcal{P}$ symmetry [28]. The bound states therefore straddle the barrier and the exchange interaction with electrons in those bound states opens an additional path for electrons to traverse the barrier. This explains the minus signs in Eqs. (6) and (7). The resulting enhancement of the barrier transmission $t$ is of order $r_{\mathrm{s}} l$ and can easily exceed the exponentially small noninteracting $t \propto \exp (-\tilde{\chi})$, which implies the exponential renormalization of $\chi$, Eq. (7).

This exponential renormalization limits our RG analysis to $\tilde{r}_{\mathrm{s}} \ll 3 \pi \exp (-\tilde{\chi})$. Also, the bound state energies $\varepsilon^{\mathrm{b}}= \pm v k_{y} \operatorname{sech} \chi$ are exponentially small at $\chi \gg 1$. The exponential renormalization of $\chi$ is thus cut-off at $\kappa^{\mathrm{b}}=\left.k T \cosh \chi\right|_{k^{\prime}=\kappa^{\mathrm{b}}} / v$ and it requires exponentially low temperatures $k T \ll \exp (-\tilde{\chi}) v / a$. Eq. (7) is solved by

$$
\chi=-\ln \left(e^{-\tilde{\chi}}+\frac{4}{3 \pi} y\right),
$$

where $y$ is evaluated at $\kappa^{\mathrm{b}}, y=\ln \left[1-\left(\tilde{r}_{\mathrm{s}} / 4\right) \ln \kappa^{\mathrm{b}} a\right]$. The self-consistent solution of Eq. (8) with this $\chi$-dependent $y$ determines the normalized conductance, which for $\chi \gg 1$ takes the form $G_{\mathrm{r}}=8 \exp (-2 \chi) / 3$. In analogy with the Kondo problem we define the temperature scale $T^{*}$ where the transmission across the barrier becomes of order unity, $G_{\mathrm{r}} \simeq 1$. At $\tilde{r}_{\mathrm{s}} \ll 3 \pi \exp (-\tilde{\chi})$ we find

$$
k T^{*}=\frac{v}{a} e^{-3 \pi / \tilde{r}_{\mathrm{s}}} .
$$

At wavevectors $k^{\prime}<\kappa^{\mathrm{b}}$ the bound states do not renormalize $\chi$ anymore, but the propagating states continue to do so and $d \chi / d y=3 / 2$ for $\kappa^{\mathrm{b}} \gg k^{\prime} \gg k T / v$. This now decreases the conductance, by a factor $\left(1+\left.\tilde{r}_{\mathrm{s}} \chi\right|_{k^{\prime}=\kappa^{\mathrm{b}}} / 4\right)^{-3}$ if $\left.\chi\right|_{k^{\prime}=\kappa^{\mathrm{b}}} \gg 1$. In Fig. 2 we plot the normalized resistance $G_{\mathrm{r}}^{-1}$ for various parameter values. Some of them are within the regime $\tilde{r}_{\mathrm{s}} \ll 3 \pi \exp (-\tilde{\chi})$, where Eq. (8) is rigorously justified, others at $\tilde{r}_{\mathrm{s}} \lesssim 3 \pi \exp (-\tilde{\chi})$, where Eq. (8) may still be qualitatively correct. At $\tilde{r}_{\mathrm{s}}>3 \pi \exp (-\tilde{\chi})$ Eq. (8) does not have a unique solution anymore. Clearly, the predicted effect is large even for moderate $\tilde{\chi} \gtrsim 1$ and $\ln \kappa^{\mathrm{b}} a \gtrsim 1$. Its observation therefore does not require access to large temperature intervals.

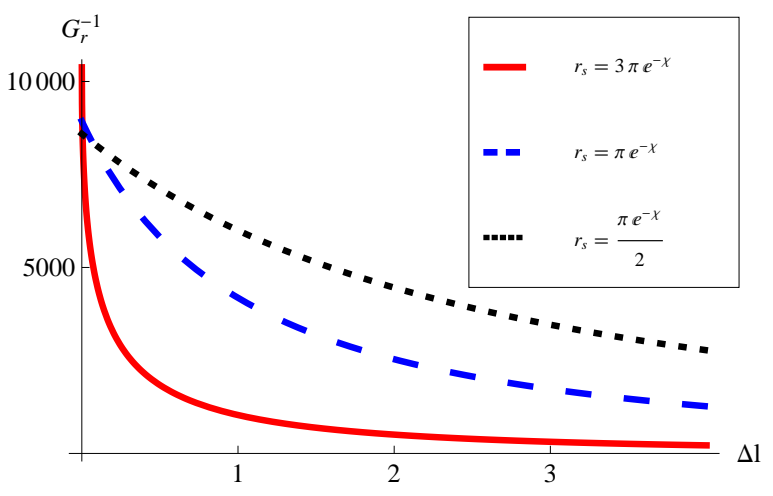

FIG. 2: Normalized resistance $G_{\mathrm{r}}^{-1}$ at $\tilde{\chi}=5$ and $k T<$ $v \operatorname{sech} \tilde{\chi} / a$, when it is renormalized by bound states, as a function of $\Delta l=\ln (v \operatorname{sech} \tilde{\chi} / k T a)$.

As $\chi$ decreases the scaling behavior crosses over into a second regime, where $\chi \ll 1$ and $F(\chi)$ may be expanded around $\chi=0$. In that case we find $d \chi / d y=-4(10 / 3 \pi-$ 1) $\chi^{3}$ [29] such that, now assuming for simplicity $\tilde{\chi} \ll 1$,

$$
\chi=\frac{\tilde{\chi}}{\sqrt{1+8 \tilde{\chi}^{2}(10 / 3 \pi-1) y}} .
$$

We conclude that the backscattering from $\mathbf{A}$ disappears asymptotically at low $T$, very differently from static scattering potentials $v$ in $1 \mathrm{D}$. The coupling $\chi$, however, is only marginally irrelevant here $\left(d \chi / d l \propto \chi^{3}[30]\right)$. It is renormalized more slowly than $v$ in $1 \mathrm{D}$, which is relevant in the RG sense $(d v / d l \propto v)$ [1]. The renormalization of $r_{\mathrm{s}}$ in graphene further slows down the RG flow of $\chi$.

Discussion: In reality the unscreened and ballistic segments of graphene around the barrier in Fig. 11 have a finite length $L$ (typically limited by contacts or by the elastic mean free path). Our predictions then require that the 
entire Friedel oscillation fits into those segments, implying an additional cut-off $\kappa^{L}=\operatorname{coth} \tilde{\chi} / L$ for the RG-flow and necessitating $L \gg a$. Due to the strong renormalization at $\chi \gg 1$, however, the observation of the effects shown in Fig. 2 does not require excessively long samples.

While inelastic processes are negligible in the above analysis of the renormalization of $\chi$ at $r_{\mathrm{s}} \ll 1$ [19], they can significantly affect the transport between barrier and contacts [20]. There inelastic scattering can be neglected only if the barrier is more resistive than the adjacent ballistic regions, such that $\left(k T G_{\mathrm{r}}\right)^{-1} \gg r_{\mathrm{s}}^{2} L / v$. The cut-off $\kappa^{L}$ further limits the regime where our predictions can be observed directly in the conductance to $r_{\mathrm{s}}^{2} G_{\mathrm{r}} \ll v / k T L \ll \tanh \tilde{\chi}$. At $r_{\mathrm{s}}^{2} G_{\mathrm{r}} \gtrsim v / k T L$ the observation of $G_{\mathrm{r}}^{-1}$ requires experimental differentiation from the inelastic background resistance, e.g. by varying $\tilde{\chi}$.

The vector potential $\mathbf{A}$ is induced by strain in the $x$ direction if that is along the "armchair" direction of the graphene lattice [17]. Strain, however, also generates a scalar, "deformation" potential $V \lesssim \tilde{\chi} v / a$ [17]. This breaks PH-symmetry, a Hartree-potential appears, and our predictions require $V \ll \max \{v \operatorname{coth} \tilde{\chi} / L, k T\} / r_{\mathrm{s}}$. Also the bound states are strongly affected by $V$ and consequently Eq. (7) holds only if $V \ll \tilde{\chi} \exp (-\tilde{\chi}) v / a$, a tight constraint when $\tilde{\chi} \gg 1$. We remark that Eq. (8) (strictly valid at $\tilde{\chi} \gg 1$, but possibly qualitatively correct also at $\tilde{\chi} \simeq 1$ ) predicts strong effects like those of Fig. 2 down to $\tilde{\chi} \simeq 1$, when this extra constraint disappears. To avoid a nonzero $V, \mathbf{A}$ can be generated alternatively by a pair of wires at $x=0$, but different distances $d_{1}, d_{2} \simeq a$ from the graphene plane (see Fig. 1). Opposite currents $I$ and $-I$ through such wires create a vector potential that falls off as $1 / x^{2}$ at $x \gg a$ and that has integral $\tilde{\chi}=\int d x A_{\mathrm{y}}(x)=\mu_{0} I\left(d_{1}-d_{2}\right)$. In our limit $r_{\mathrm{s}} \ll 1$, but $l \gg 1$ the deviations of this potential from the square-well shape Eq. (1) have negligible effects.

Conclusions: We have shown that the Coulomb repulsion between electrons can have profound and unconventional effects on transport in intrinsic graphene. General considerations predict singular transport across energyindependent $1 \mathrm{D}$ defects without Hartree potential. In addition we have shown that interactions in graphene can enhance transport through static scatterers, in surprising contrast with the familiar result for $1 \mathrm{D}$ conductors. We have presented detailed calculations for vector potential scatterers that are induced by strain. There the above effects have exponentially large experimental signatures.

The author thanks Yu. V. Nazarov for discussion and the KITP at UCSB for hospitality, where this work was supported in part by the NSF, Grant No. PHY05-51164.

[1] C. L. Kane and M. P. A. Fisher, Phys. Rev. B 46, 15233 (1992).

[2] K. A. Matveev, D. Yue, and L. I. Glazman, Phys. Rev. Lett. 71, 3351 (1993).

[3] D. Yue, L. I. Glazman, and K. A. Matveev, Phys. Rev. B 49, 1966 (1994).
[4] B. Altshuler and A. Aronov, in Electron-Electron Interactions in Disordered Systems, North-Holland, Amsterdam (1985).

[5] G. Zala, B. N. Narozhny, and I. L. Aleiner, Phys. Rev. B 64, 214204 (2001).

[6] V. V. Cheianov and V. I. Fal'ko, Phys. Rev. Lett. 97, 226801 (2006).

[7] L. Shekhtman and L. I. Glazman, Phys. Rev. B 52, R2297 (1995).

[8] A. Y. Alekseev and V. V. Cheianov, Phys. Rev. B 57, R6834 (1998).

[9] K. Novoselov, A. Geim, S. Morozov, D. Jiang, Y. Zhang, S. Dubonos, I. Grigorieva, and A. Firsov, Science 306, 666 (2004).

[10] Y. Zhang, Y.-W. Tan, H. L. Stormer, and P. Kim, Nature 438, 201 (2005).

[11] C. Berger, Z. Song, T. Li, X. Li, A. Y. Ogbazghi, R. Feng, Z. Dai, A. N. Marchenkov, E. H. Conrad, P. N. First, et al., J. Phys. Chem. B 108, 19912 (2004).

[12] J. González, F. Guinea, and M. A. H. Vozmediano, Phys. Rev. B 59, R2474 (1999).

[13] Z. Li, E. Henriksen, Z. Jiang, Z. Hao, M. Martin, P. Kim, H. Stormer, and D. Basov, Nature Physics 4, 532 (2008).

[14] Y. Barlas, T. Pereg-Barnea, M. Polini, R. Asgari, and A. H. MacDonald, Phys. Rev. Lett. 98, 236601 (2007).

[15] T. Stauber, F. Guinea, and M. A. H. Vozmediano, Phys. Rev. B 71, 041406(R) (2005).

[16] M. S. Foster and I. L. Aleiner, Phys. Rev. B 77, 195413 (2008).

[17] M. M. Fogler, F. Guinea, and M. I. Katsnelson, Phys. Rev. Lett. 101, 226804 (2008).

[18] V. M. Pereira and A. H. C. Neto, Phys. Rev. Lett. 103, 046801 (2009).

[19] M. P. A. Fisher and L. I. Glazman, in Mesoscopic Electron Transport, ed. L. Kouwenhoven, G. Schön, and L. Sohn, NATO ASI E, Kluwer Ac. Publ., Dordrecht (1996).

[20] L. Fritz, J. Schmalian, M. Müller, and S. Sachdev, Phys. Rev. B 78, 085416 (2008).

[21] E. H. Hwang and S. Das Sarma, Phys. Rev. B 75, 205418 (2007).

[22] B. Wunsch, T. Stauber, F. Sols, and F. Guinea, New J. Phys. 8, 318 (2006).

[23] Staggered potentials do have singular interaction corrections [15, 16], but they require atomically sharp disorder.

[24] In the case of PH symmetry this is seen easiest in second quantized form, when $H$ is invariant under the transformation $c \rightarrow \sigma_{z} c^{\dagger}$, where $c$ is the electron annihilation operator. $\mathrm{PH}$-symmetry requires vanishing chemical potential and interaction with the ion charge density.

[25] Screening by the electrons in the graphene sheet itself is negligible at the wavevectors $k^{\prime} \gg k T / v$ that make the dominant contributions to the analyzed effects 21, 22].

[26] In case $\mathbf{A}$ is due to strain, $\tilde{\chi}$ has opposite sign in the two valleys [17]. Because of the invariance of transport under $\tilde{\chi} \rightarrow-\tilde{\chi}$, however, also this does not affect our results.

[27] $G_{\mathrm{r}}$ is measured best when $\mathbf{A}$ is not due to strain, but electric currents that can easily be switched on and off.

[28] The bound states are non-degenerate (apart from the spin and valley degeneracies).

[29] This equation has corrections of order $\chi k^{\prime} a$. It is strictly valid for $k^{\prime} a \ll \tilde{\chi}$ (e.g. when gates around $x=0$ screen short wavelength Friedel oscillations), or at $1 / \tilde{\chi}^{2} y \ll 1$.

[30] The absence of a term $\propto \chi$ follows also from Refs. [15, 16]. 